\title{
On the Role Played by the Extensional Behavior of Giesekus Fluids in Plane Stagnation Flow
}

\author{
Mohammad MiRZADEH and Kayvan SADEGHY ${ }^{\dagger}$ \\ University of Tehran, College of Engineering, Department of Mechanical Engineering \\ P.O. Box: 11155 - 4563, Tehran, Iran \\ (Received : June 20, 2008)
}

\begin{abstract}
An exact solution has been found in plane stagnation flow for a viscoelastic fluid obeying the Giesekus model. The flow was found to render itself to a local similarity solution in the vicinity of the stagnation point. The solution so obtained could easily enable one to investigate the effects of parameters such as Reynolds number, Weissenberg number and the mobility factor on the velocity and stress fields. Unlike Newtonian and Maxwellian fluids, the thickness of the boundary layer was found to be a decreasing function of the distance from the stagnation point. It will be shown that this effect may have roots in the extensional behavior of the fluid.
\end{abstract}

Key Words: Giesekus fluid / Stagnation flow / Exact solution / Mobility factor / Extensional viscosity

\section{INTRODUCTION}

Exact solutions are rather rare in fluid mechanics and this is particularly so for non-Newtonian fluids. This is perhaps why the field of non-Newtonian fluid mechanics relies so heavily on approximate theories such as the creeping flow theory and/or the boundary layer theory. ${ }^{1-4)}$ But these approximate theories are still incomplete as far as non-Newtonian fluids are concerned..$^{1-4)}$ Thus, the significance of theoretical results obtained using such theories are in serious doubt when dealing with viscoelastic fluids such as polymeric liquids. To obtain more reliable results one has no choice other than keeping all viscous, inertia, and elastic terms in the equations of motion. Unfortunately, this often leads to a formidable fluid mechanics problem which can, at best, be solved through computational methods only., ${ }^{5,6)}$ But computer codes need to be verified first before being of any good in dealing with practical fluid mechanics problems. And, ironically, this can only be done by confronting their output with an exact analytical solution at the first place. Evidently, exact solutions are indispensable tools in the field of non-Newtonian fluid mechanics.

Exact solutions are available for simple rheological models such as power-law and second-order models in simple geometries such as pipe flow. ${ }^{7,8)}$ Such exact solutions can be used, and have indeed been used in the past, to calibrate

\footnotetext{
$\uparrow$ To whom correspondence should be addressed.

E-mail: sadeghy@ut.ac.ir, Tel: +98 21 61114011, Fax: +98 2188013029
}

computer codes. However, one should always bear in mind that a computer code which behaves decently in simple geometries such as Poiseuille flow may fail rather miserably in complex geometries such as Jeffrey-Hamel flow. This means that code verification should preferably be done using exact solutions found in non-trivial flows (e.g., converging/ diverging flow, rotating-disk flow, stagnation-point flow, and the like). And, this should preferably be done using rheological models which can better represent viscoelastic fluids such as polymeric liquids. Fluid models for which a couple of non-trivial exact solutions have been found in the past are the Maxwell model and the Oldroyd-B model. ${ }^{9-15)}$

Among non-trivial exact solutions previously found for Maxwell and Oldroyd-B models, the stagnation-point flow is of particular interest in that it is one of the few geometries in which fluid elements are subjected to strong extensional deformations in addition to shear deformations (see Fig. 1). Further interest in this particular flow arises from the fact that it constitutes the starting point in many boundary layer studies related to blunt bodies such as torpedoes and projectiles. For reasons like these, stagnation flows are regarded as one of the best benchmark fluid mechanics problems for checking the performance of available, or under development, computer codes. Unfortunately, the range of applicability of exact solutions found for Maxwell and Oldroyd-B models is quite limited. This is because these two fluid models cannot account for shear-dependent viscosity of, say, polymeric liquids. Nor can they predict a finite extensional viscosity for such 
fluids in steady uniaxial extension. ${ }^{16,17)}$ Thus a computer code calibrated using these exact solutions may render unrealistic results when applied to flows governed by strong elongational deformations

To remedy the shortcomings of the Maxwell and Oldroyd-B models in studies related to the flow of viscoelastic fluids, use should preferably be made of more realistic fluid models such as the Giesekus model, the Phan-Thien-Tanner model, the Leonov model, and the Johnson-Segalman model. ${ }^{16,17)}$ In the present work, an exact solution will be reported (to the best of our knowledge for the first time) for Giesekus fluids in plane stagnation flow. This particular fluid model has been selected for the present study because of its excellent performance in shear flows and its good performance in extensional flows. As such, an exact solution found for this particular rheological model in stagnation-point flow can be argued to be far more superior to those previously found for Maxwell and Oldroyd-B models. From a more fundamental point of view, the solution so obtained can enable one to investigate the role played by the extensional behavior of a fluid on the characteristics of its boundary layer in stagnation flows.

\section{THEORY}

Consider two-dimensional, laminar flow of an incompressible fluid in the vicinity of a stagnation point under steady and isothermal flow conditions (see Fig. 1).

In Cartesian coordinate system, the equations governing the flow are the continuity equation and the two momentum equations:

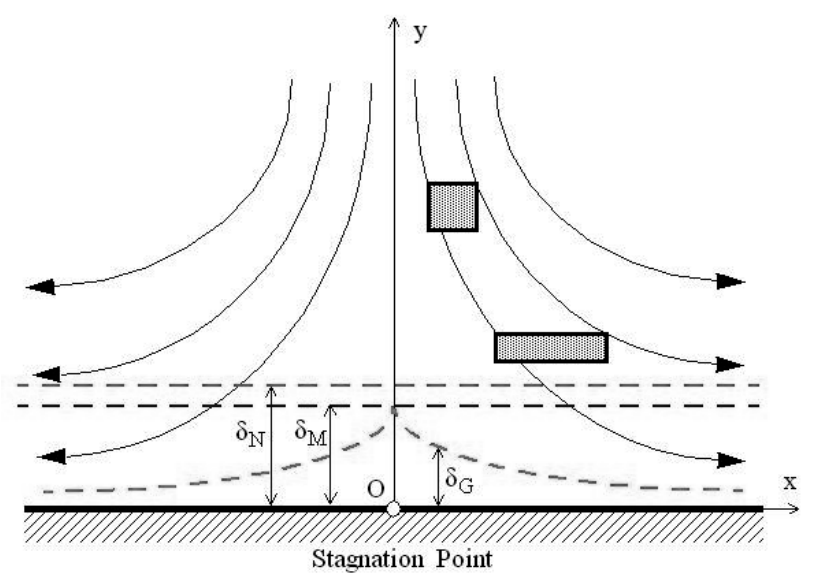

Fig. 1. Schematic showing the elongational effect of stagnation-point flows on fluid elements $\left(\delta_{\mathrm{N}}, \delta_{\mathrm{M}}\right.$, and $\delta_{\mathrm{G}}$ are the thickness of the boundary layer for Newtonian, Maxwellian and Giesekus fluids, respectively).

$$
\begin{aligned}
& \frac{\partial u}{\partial x}+\frac{\partial v}{\partial y}=0 \\
& \rho\left(u \frac{\partial u}{\partial x}+v \frac{\partial u}{\partial y}\right)=-\frac{\partial p}{\partial x}+\frac{\partial \tau_{x x}}{\partial x}+\frac{\partial \tau_{x y}}{\partial y} \\
& \rho\left(u \frac{\partial v}{\partial x}+v \frac{\partial v}{\partial y}\right)=-\frac{\partial p}{\partial y}+\frac{\partial \tau_{y x}}{\partial x}+\frac{\partial \tau_{y y}}{\partial y}
\end{aligned}
$$

We assume that as $y$ goes to infinity a potential flow, identical to that for Newtonian fluids, is recovered; that is,

$$
\text { as } \mathrm{y} \rightarrow \infty ; \mathrm{u} \rightarrow \gamma \mathrm{x} ; \mathrm{v} \rightarrow-\gamma \mathrm{y}
$$

where $\gamma$ is a constant related to the upstream conditions having the dimension of a shear rate. For a fluid obeying the Giesekus model as its constitutive equation, the extra stress tensor is given by ${ }^{16,17)}$

$$
\tau+\frac{\lambda \alpha}{\mu} \tau \cdot \tau+\lambda \stackrel{\nabla}{\tau}=\mu\left[\nabla \mathbf{V}+(\nabla \mathbf{V})^{T}\right]
$$

where $\nabla$ refers to the upper-convected time derivative, $\mathbf{V}$ is the velocity vector, $\mu$ is the viscosity coefficient, $\alpha$ is the mobility parameter, and $\lambda$ is the relaxation time of the fluid, with the superscript " $T$ " denoting a transpose.

The Giesekus model is regarded as one of the best rheological models for representing polymeric liquids. This is because it correctly predicts shear-thinning, non-vanishing first and second normal stress differences, a finite extensional viscosity, and a non-exponential stress relaxation or stress growth curve for polymeric liquids. The mobility factor, $\alpha$, plays a crucial role in the Giesekus model as it gives rise to a nonlinear stress term in the constitutive equation (Eq. 5). In fact, for $\alpha=0$ the Giesekus model is simply reduced to the Maxwell model-a rather unrealistic viscoelastic fluid having constant viscosity and unbounded extensional viscosity at finite extension rates. From a physical point of view, the mobility factor describes how the relaxation time of, say, a polymer macromolecule is altered when the surrounding molecules are oriented in the flow direction. In other words, it accounts for anisotropic relaxation of fluid elements with a subsequent effect on the shear and extensional behavior of the fluid. In effect, by increasing $\alpha$ from zero, shear-thinning starts at lower shear rates. Similarly, the asymptotic value of the extensional viscosity is decreased in uniaxial extension when $\alpha$ is increased from zero. The last thing remained to be said about the mobility factor is that for the Giesekus model to comply with experimental data the mobility factor should be selected in the range of $0<\alpha \leq 0.5$ regardless of the fact that theoretically it can take values up to one. ${ }^{16,17)}$ 
Now, to initiate the analysis like Phan-Thien ${ }^{13,14)}$ we express the velocity field in terms of an auxiliary function $\phi(\eta)$ such that the two velocity components satisfy the continuity equation identically; that is,

$$
\begin{aligned}
& \mathrm{u}(\mathrm{x}, \eta)=\mathrm{x} \gamma \phi^{\prime}(\eta) \\
& \mathrm{v}(\eta)=-\sqrt{\frac{\mu \gamma}{\rho}} \phi(\eta)
\end{aligned}
$$

where prime denotes differentiation with respective to $\eta$ (i.e., the similarity variable) defined by:

$$
\eta=y \sqrt{\frac{\rho \gamma}{\mu}}
$$

Having inserted the velocity field, as given by Eqs. 6 and 7, into Eq. 5, the following equations are obtained for the stress components:

$$
\begin{gathered}
\tau_{\mathrm{xx}}+\mathrm{Wi}\left(\begin{array}{l}
\frac{\alpha}{\gamma \mu}\left(\tau_{\mathrm{xx}}^{2}+\tau_{\mathrm{xy}}^{2}\right)-2 \phi^{\prime} \tau_{\mathrm{xx}}-\phi \tau_{\mathrm{xx}}^{\prime}- \\
2 \mathrm{x} \sqrt{\frac{\rho \gamma}{\mu}} \phi^{\prime \prime} \tau_{\mathrm{xy}}+\mathrm{x} \phi^{\prime} \frac{\partial \tau_{\mathrm{xx}}}{\partial \mathrm{x}}
\end{array}\right)=2 \gamma \mu \phi^{\prime} \\
\tau_{\mathrm{xy}}+\mathrm{Wi}\left(\begin{array}{l}
\frac{\alpha}{\gamma \mu}\left(\tau_{\mathrm{xx}} \tau_{\mathrm{xy}}+\tau_{\mathrm{xy}} \tau_{\mathrm{yy}}\right)-\phi \tau_{\mathrm{xy}}^{\prime}- \\
\mathrm{x} \sqrt{\frac{\rho \gamma}{\mu}} \phi^{\prime \prime} \tau_{\mathrm{yy}}+\mathrm{x} \phi^{\prime} \frac{\partial \tau_{\mathrm{xy}}}{\partial \mathrm{x}}
\end{array}\right)=\mathrm{x} \gamma \mu \sqrt{\frac{\rho \gamma}{\mu}} \phi^{\prime \prime} \\
\tau_{\mathrm{yy}}+\mathrm{Wi}\left(\begin{array}{l}
\frac{\alpha}{\gamma \mu}\left(\tau_{\mathrm{xy}}^{2}+\tau_{\mathrm{yy}}^{2}\right)+2 \phi^{\prime} \tau_{\mathrm{yy}}- \\
\phi \tau_{\mathrm{yy}}^{\prime}+\mathrm{x} \phi^{\prime} \frac{\partial \tau_{\mathrm{yy}}}{\partial \mathrm{x}}
\end{array}\right)=-2 \gamma \mu \phi^{\prime}
\end{gathered}
$$

where $\mathrm{Wi}$ is the Weissenberg number defined by $\mathrm{Wi}=\lambda \gamma$. It goes without saying that the above velocity and stress fields should satisfy the governing equations (i.e, Eqs. 2 and 3) to be of any physical significance. Interestingly, these equations are also satisfied, identically, by a stress field of the following form,

$$
\begin{aligned}
& \tau_{\mathrm{xx}}=\mu \gamma \mathrm{T}_{1}(\eta)+\rho \gamma^{2} \mathrm{x}^{2} \mathrm{~T}_{2}(\eta) \\
& \tau_{\mathrm{xy}}=\mathrm{x} \mu \gamma \sqrt{\frac{\rho \gamma}{\mu}} \mathrm{T}_{3}(\eta) \\
& \tau_{\mathrm{yy}}=\mu \gamma \mathrm{T}_{4}(\eta)
\end{aligned}
$$

For this to be true, it is required that the following relationship (having been obtained by eliminating the pressure term between Eq. 2 and Eq. 3) holds for compatibility:

$$
2 \mathrm{~T}_{2}+\mathrm{T}_{3}^{\prime}-\phi^{\prime 2}+\phi \phi^{\prime \prime}=-1
$$

To determine the unknown functions $\mathrm{T}_{1}, \mathrm{~T}_{2}, \mathrm{~T}_{3}$, and $\mathrm{T}_{4}$, the stress terms as given by Eqs. 12-14 will be inserted into Eqs. 9-11. The resulting equations are

$$
\begin{aligned}
& \mathrm{T}_{1}+\mathrm{Wi}\left(\alpha \cdot \mathrm{T}_{1}^{2}-2 \phi^{\prime} \mathrm{T}_{1}-\phi \mathrm{T}_{1}^{\prime}\right)-2 \phi^{\prime}=0 \\
& \mathrm{~T}_{2}+\mathrm{Wi}\left[\alpha \cdot\left(\mathrm{T}_{3}^{2}+2 \mathrm{~T}_{1} \mathrm{~T}_{2}+\mathrm{Re}_{\mathrm{x}} \mathrm{T}_{2}^{2}\right)\right. \\
& \left.-\phi \mathrm{T}_{2}^{\prime}-2 \phi^{\prime \prime} \mathrm{T}_{3}\right]=0 \\
& \mathrm{~T}_{3}+\mathrm{Wi}\left[\alpha \cdot\left(\mathrm{T}_{3} \mathrm{~T}_{4}+\mathrm{T}_{1} \mathrm{~T}_{3}+\operatorname{Re}_{\mathrm{x}} \mathrm{T}_{2} \mathrm{~T}_{3}\right)-\right. \\
& \left.\phi \mathrm{T}_{3}^{\prime}+\phi^{\prime} \mathrm{T}_{3}-\phi^{\prime \prime} \mathrm{T}_{4}\right]-\phi^{\prime \prime}=0 \\
& \mathrm{~T}_{4}+\mathrm{Wi}\left[\alpha \cdot\left(\mathrm{T}_{4}^{2}+\mathrm{Re}_{\mathrm{x}} \mathrm{T}_{3}^{2}\right)-\right. \\
& \left.\phi \mathrm{T}_{4}^{\prime}+2 \phi^{\prime} \mathrm{T}_{4}\right]+2 \phi^{\prime}=0
\end{aligned}
$$

where $\operatorname{Re}_{x}$ is the local Reynolds number defined by $\operatorname{Re}_{\mathrm{x}}=\rho \mathrm{x}^{2} \gamma / \mu$. From Eqs. 16-19 it can be concluded that unlike Maxwell and Oldroyd-B fluids ${ }^{13,14)}$, Giesekus fluids do not render themselves to a self-similar solution in plane stagnation flow because the $\mathrm{x}$-coordinate cannot be eliminated from these equations. The lack of a self-similar solution for Giesekus fluids in stagnation flow can be attributed to the mobility factor because by setting $\alpha=0$ the x-coordinate (which appears in $\mathrm{Re}_{x}$ ) is completely eliminated from Eqs. 17-19. As mentioned above, the mobility dramatically affects shear and extensional flow behavior of Giesekus fluids. Since purelyviscous shear-thinning fluids (e.g., fluids obeying the powerlaw model) are known to render themselves to a self-similar solution in stagnation flow ${ }^{18)}$, thus the lack of a self-similar solution for Giesekus fluids in stagnation flows may have roots in the extensional behavior of the fluid-a conjecture which will be elaborated more in the subsequent sections.

With no self-similar solution available, we will try to find a local similarity solution for the problem at hand. Such a solution, if it exists, would enable one to investigate the effects of parameters such as the Weissenberg number and/or the mobility factor on the velocity and stress profiles at any given $\mathrm{x}$-location sufficiently close to the stagnation point. To achieve this goal, one has to solve Eqs. 15-19 for the unknown functions $\mathrm{T}_{1}, \mathrm{~T}_{2}, \mathrm{~T}_{3}, \mathrm{~T}_{4}, \phi$ at any given $\mathrm{x}$-location. The boundary conditions required to solve these equations are the no-slip condition at the wall, the wall being impermeable, and the velocity components to approach the potential flow velocity field (see Eq. 4); that is,

$$
\phi(0)=\phi^{\prime}(0)=0 ; \phi^{\prime}(\infty)=1
$$


As to the stress components $\tau_{x x}, \tau_{y y}, \tau_{x y}$, we assume that by approaching infinity, they all become constant. This means that at infinity we have $\mathrm{T}_{1}^{\prime}(\infty)=\mathrm{T}_{2}^{\prime}(\infty)=\mathrm{T}_{3}^{\prime}(\infty)=\mathrm{T}_{4}^{\prime}(\infty)=0$. Knowing $\mathrm{T}_{1}^{\prime}, \mathrm{T}_{2}^{\prime}, \mathrm{T}_{3}^{\prime}, \mathrm{T}_{4}^{\prime}$, and $\phi^{\prime}$ at infinity, we can write down Eqs. 15-19 at infinity and solve them for $\mathrm{T}_{1}, \mathrm{~T}_{2}, \mathrm{~T}_{3}, \mathrm{~T}_{4}$. By so doing, the boundary conditions on $\mathrm{T}_{1}, \mathrm{~T}_{2}, \mathrm{~T}_{3}, \mathrm{~T}_{4}$ at infinity become:

$$
\begin{aligned}
& \mathrm{T}_{1}(\infty)=\frac{2 \mathrm{Wi}-1+\sqrt{\mathrm{A}}}{2 \alpha \mathrm{Wi}} \\
& \mathrm{T}_{2}(\infty)=\mathrm{T}_{3}(\infty)=0 \\
& \mathrm{~T}_{4}(\infty)=\frac{-(2 \mathrm{Wi}+1)+\sqrt{\mathrm{B}}}{2 \alpha \mathrm{Wi}}
\end{aligned}
$$

where,

$$
\begin{aligned}
& \mathrm{A}=(2 \mathrm{Wi}-1)^{2}+8 \alpha \mathrm{Wi} \\
& \mathrm{B}=(2 \mathrm{Wi}+1)^{2}-8 \alpha \mathrm{Wi}
\end{aligned}
$$

\section{METHOD OF SOLUTION}

The governing equations, Eqs. 15-19, subject to the boundary conditions, Eqs. 20 and 21, look too formidable to render themselves to an analytical solution. Thus, we have decided to resort to the Keller's Box method in order to find a tractable numerical solution. The Keller's Box is among implicit methods with a second-order accuracy in space and time. ${ }^{19)}$ The scheme is good in that it allows non-uniform grids to be used near the wall making it possible to better resolve the thin boundary layer near the wall. The Keller's Box scheme has the further advantage that it deals with firstorder derivatives only. This property facilitates numerical manipulation as well as implementation of the boundary conditions. As a drawback, the size of the coefficient matrix is increased due to the emergence of new unknowns. In the Keller's Box method all derivatives are evaluated at the midpoint grid, and all non-derivative terms are calculated using an averaging rule at the same point. That is, for an arbitrary function $\mathrm{F}$ we have:

$$
\begin{aligned}
& \left(F_{j}^{\prime}\right)_{i-1 / 2}=\frac{\left(F_{j}\right)_{i}-\left(F_{j}\right)_{i-1}}{h_{i}} ; \\
& \left(F_{j}\right)_{i-1 / 2}=\frac{\left(F_{j}\right)_{i}+\left(F_{j}\right)_{i-1}}{2}
\end{aligned}
$$

Due to the non-linear nature of the governing equations, a linearization technique must be invoked in the solution scheme. In this study, Newton's linearization method will be used for this purpose. To that end, we write $\left(\mathrm{F}_{\mathrm{j}}^{\mathrm{n}+1}\right)_{\mathrm{i}}=\left(\mathrm{F}_{\mathrm{j}}^{\mathrm{n}}\right)_{\mathrm{i}}+\mathrm{k}\left(\mathrm{F}_{\mathrm{j}}\right)_{\mathrm{i}}$, where $k$ is a correcting factor. Now, by introducing Eq. 23 into the governing equations, an algebraic matrix relationship is obtained for the correcting factor. Like any other iterative method, one needs an initial guess to initiate the solution. With an eye on the boundary conditions at the wall and also at infinity, we use the following initial guess for this purpose:

$$
\begin{aligned}
& \widetilde{\phi}_{\mathrm{i}}=0 ; \widetilde{\varphi}_{\mathrm{i}}=\frac{\mathrm{i}-1}{\mathrm{~N}-1} \\
& \widetilde{\mathrm{T}}_{1_{\mathrm{i}}}=\frac{2 \mathrm{Wi}-1+\sqrt{\mathrm{A}}}{2 \alpha \mathrm{Wi}} \\
& \widetilde{\mathrm{T}}_{2_{\mathrm{i}}}=0 ; \widetilde{\mathrm{T}}_{3_{\mathrm{i}}}=0 \\
& \widetilde{\mathrm{T}}_{4_{\mathrm{i}}}=\frac{-2 \mathrm{Wi}-1+\sqrt{\mathrm{B}}}{2 \alpha \mathrm{Wi}}
\end{aligned}
$$

where $N$ is the total number of grid points. Once the correcting factors are determined, the solution can easily be updated to the next step. The whole process is then repeated until the correcting factors are less than a preset small value $\left(\right.$ say, $10^{-10}$ ).

\section{RESULTS AND DISCUSSIONS}

Before using the computer code developed in the present work to calculate the quantities of interest for Giesekus fluids in stagnation flow, the code was validated first using known data for Newtonian and Maxwellian fluids. From the classical work of Hiemenz ${ }^{20)}$ on Newtonian fluids, the ratio $C_{f} / 2 \sqrt{\operatorname{Re}_{x}}$ (where $C_{f}$ is the skin friction coefficient) is known to be equal to 1.2326 for these fluids. The code developed in this work yields a value of 1.2325 for this ratio demonstrating that it works perfectly as far as Newtonian fluids are concerned.

As to the Maxwellian fluids (i.e., for $\alpha=0$ ), Fig. 2 shows that our numerical results obtained at a typical Weissenberg number of $W i=0.2$ are virtually the same as those reported by Phan-Thien ${ }^{13)}$ demonstrating that the code is good also for viscoelastic fluids. To further substantiate this, we have decided to compare our numerical results for the boundary layer thickness (defined as the point at which $\phi^{\prime}=0.99$ ) with those reported by Phan-Thien. ${ }^{13)}$ As can be seen in Table I, the two sets of data are virtually the same.

Having validated the code, it was then used to investigate the effects of parameters such as Wi, Re, and $\alpha$ on the velocity profiles for a Giesekus fluid in plane stagnation flow. Figure 3 shows the effect of the Weissenberg number, $W i$, on the velocity profiles at a typical mobility factor of $\alpha=0.5$ and Reynolds number of $\operatorname{Re}_{\mathrm{x}}=0.5$. This figure also includes the case of $\mathrm{Wi}=0$ (i.e., the Newtonian case) for comparison purposes. From this figure it can be concluded 
that, in stagnation-point flow the boundary layer is thinner for viscoelastic fluids as compared to Newtonian fluids. The boundary layer is also predicted to become thinner the larger the fluid's elasticity (as represented by the Weissenberg number, Wi). To show this in a more quantitative term, we have prepared Table II which shows the effect of Wi on the boundary layer thickness for different values of the mobility factor. At a given $\mathrm{x}$-location (corresponding to a given $\mathrm{Re}_{\mathrm{x}}$ ), the boundary layer is seen to become thinner the higher the Weissenberg number.

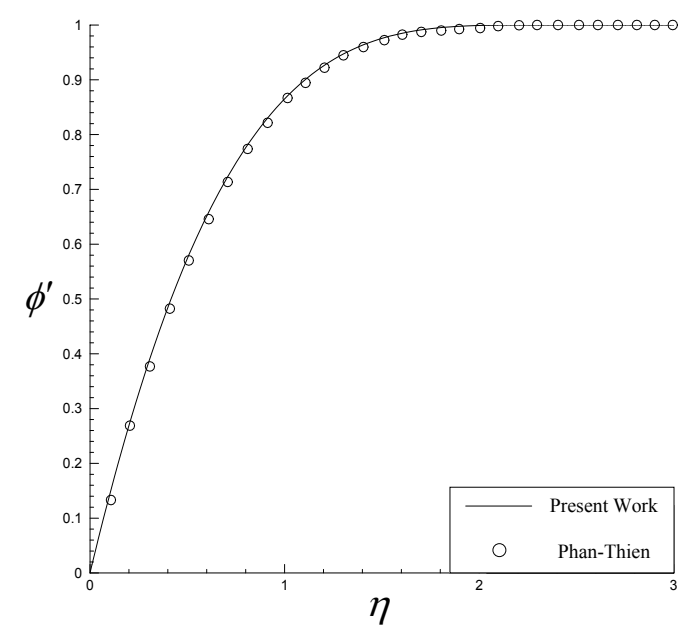

Fig. 2. A comparison between velocity profiles obtained in the present work for plane stagnation flow of Maxwellian fluids $(\alpha=0)$ with those published in Ref. 13.

Table I. A comparison between the boundary layer thickness obtained in the present work for Maxwell fluids with those reported by PhanThien. ${ }^{13)}$

\begin{tabular}{ccc}
\hline$W i$ & $\begin{array}{c}\text { Maxwell Model } \\
\text { (Phan-Thien) }\end{array}$ & $\begin{array}{c}\text { Maxwell Model } \\
\text { (Present Work) }\end{array}$ \\
\hline 0.1 & 1.980 & 1.971 \\
0.2 & 1.690 & 1.679 \\
0.3 & 1.470 & 1.458 \\
0.4 & 1.300 & 1.288 \\
\hline
\end{tabular}

Table II. The effect of Weissenberg number on dimensionless boundary layer thickness in stagnation flows of Giesekus fluids at different mobility factors.

\begin{tabular}{ccccc}
\hline \multirow{2}{*}{$\boldsymbol{W} i$} & \multicolumn{4}{c}{ Giesekus Model } \\
\cline { 2 - 5 } & $\alpha=0.1$ & $\alpha=0.3$ & $\alpha=0.5$ & $\alpha=0.7$ \\
\hline 0.1 & $\delta=1.964$ & $\delta=1.956$ & $\delta=1.952$ & $\delta=1.951$ \\
0.2 & $\delta=1.655$ & $\delta=1.623$ & $\delta=1.601$ & $\delta=1.584$ \\
0.3 & $\delta=1.416$ & $\delta=1.360$ & $\delta=1.317$ & $\delta=1.269$ \\
0.4 & $\delta=1.222$ & $\delta=1.157$ & $\delta=1.092$ & $\delta=0.986$ \\
\hline
\end{tabular}

For Maxwell fluids, the thinning effect of the fluid's elasticity on the boundary layer thickness can be attributed to the accelerating effect of $\tau_{\mathrm{xx}}$. This argument can also be applied to the Giesekus fluids. That is, as shown in Fig. 4, at any give $\mathrm{x}$-location $\tau_{\mathrm{xx}}$ is increased for Giesekus fluids when the Weissenberg number is increased. But unlike Maxwell fluids for which $\tau_{y y}$ is zero, for Giesekus fluids $\tau_{y y}$ is nonzero and negative. This means that, like $\tau_{\mathrm{xx}}$, the effect of $\tau_{\mathrm{yy}}$ on fluid elements is also accelerative. Thus, it can be argued that it is the first normal difference, $\tau_{x x}-\tau_{y y}$, which is the driving force behind fluid acceleration. In fact, as shown in Fig. 5 , by an increase in the Weissenberg number, $\tau_{x x}-\tau_{y y}$ is significantly increased. Thus it should not be surprising that at corresponding Weissenberg numbers the boundary layer at

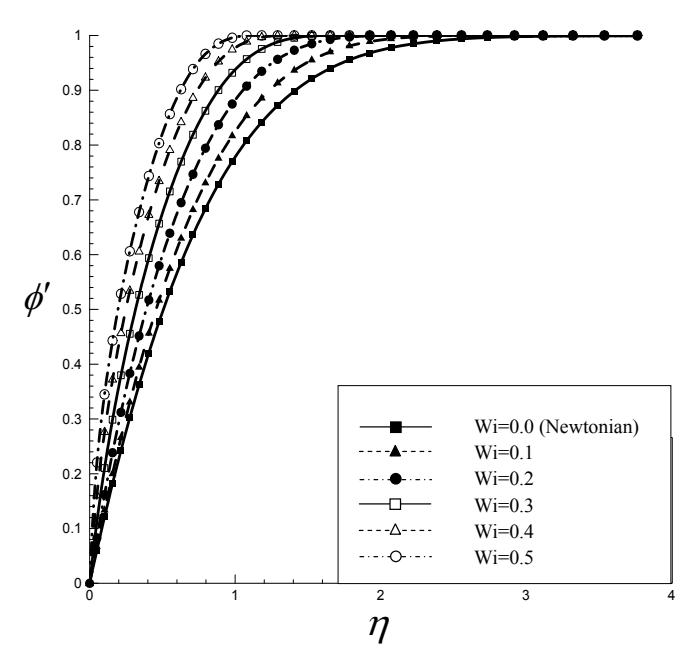

Fig. 3. The effect of the Weissenberg number on the velocity profiles for a Giesekus fluid in plane stagnation flow $\left(\alpha=0.25, \operatorname{Re}_{\mathrm{x}}=5\right)$.

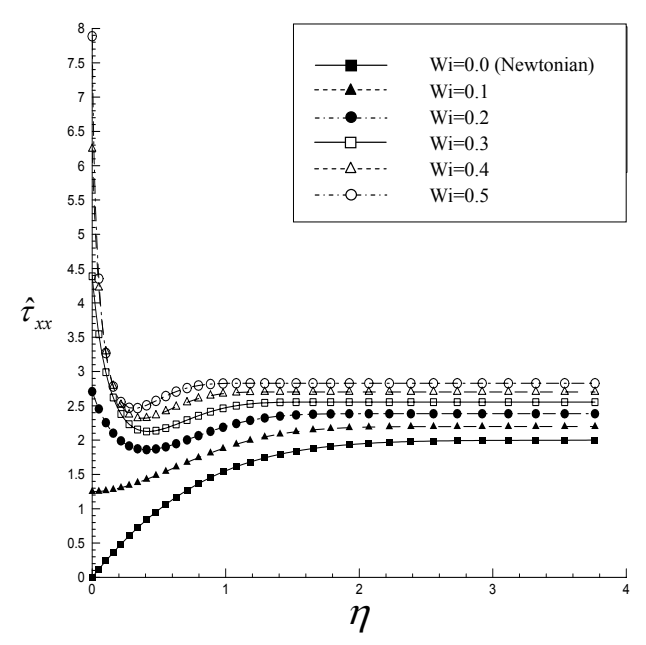

Fig. 4. The effect of the Weissenberg number on the dimensionless primary normal stress $\hat{\tau}_{x x}$ for a Giesekus fluid in plane stagnation flow $\left(\alpha=0.25, \operatorname{Re}_{\mathrm{x}}=5\right)$. 
any given $\mathrm{x}$-location becomes thinner for Giesekus fluids as compared to the Maxwell fluids.

Another quantity of interest in stagnation flow is the variation of the boundary layer thickness along the wall. For Newtonian and Maxwellian fluids, the boundary layer formed in the vicinity of the stagnation point is known to have a constant thickness. This can be attributed to a balance between the convection of vorticity along the wall and its diffusion away from the wall. To see if this is also true for Giesekus fluids, in Fig. 6 we have plotted the velocity profiles at different $\mathrm{x}$-locations (or, equivalently, at different Reynolds numbers, $\mathrm{Re}_{\mathrm{x}}$ ) along the wall. The boundary layer thickness, as calculated from these figures, has been presented in Fig. 7.

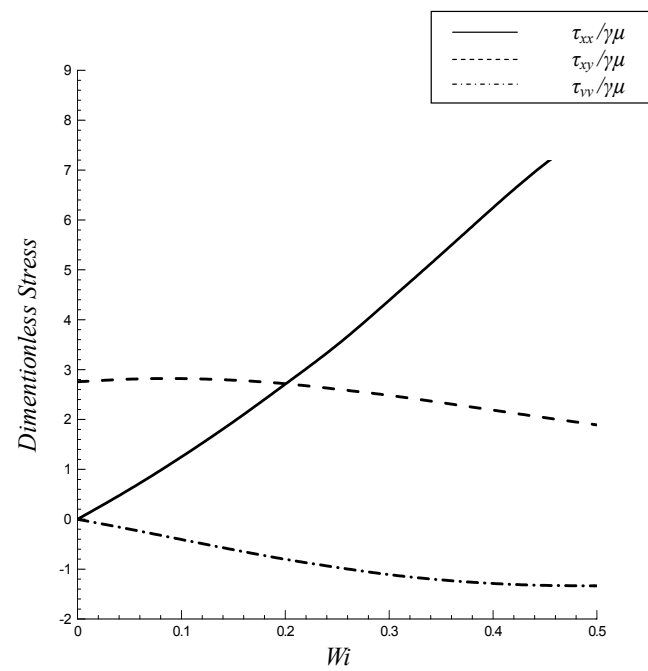

Fig. 5. The effect of the Weissenberg number on the three stress components calculated at the wall for a Giesekus fluid in plane stagnation flow $\left(\alpha=0.25\right.$ and $\left.\operatorname{Re}_{\mathrm{x}}=5\right)$.

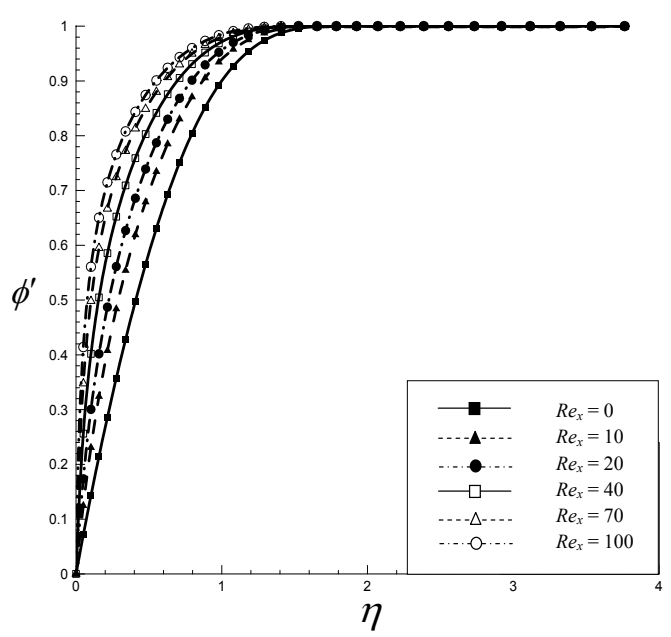

Fig. 6. The effect of the Reynolds number on the velocity profiles for a Giesekus fluid in plane stagnation flow $(\alpha=0.25, \mathrm{Wi}=0.1)$
This figure clearly shows that for Giesekus fluids the thickness of the boundary layer is a decreasing function of the distance from the stagnation point (see, also, Fig. 1). This effect can again be attributed to the accelerating effect of $\tau_{x x}-\tau_{y y}$ on the fluid elements close to the wall. As can be inferred from Figs 8 and 9, for fluid elements which lie within the boundary layer, $\tau_{\mathrm{xx}}-\tau_{\mathrm{yy}}$ increases in the $\mathrm{x}$-direxction. To better appreciate the accelerating effect of $\tau_{x x}-\tau_{y y}$, one should note that the shear stress experienced by fluid elements decreases adjacent to the wall in the x-direction, as shown in Fig. 10.

Figure 11 shows the effect of the mobility factor on the velocity profiles at a typical x-location corresponding to $\operatorname{Re}_{x}=5$. Although this figure includes results for $\alpha$ up to 0.75 ,

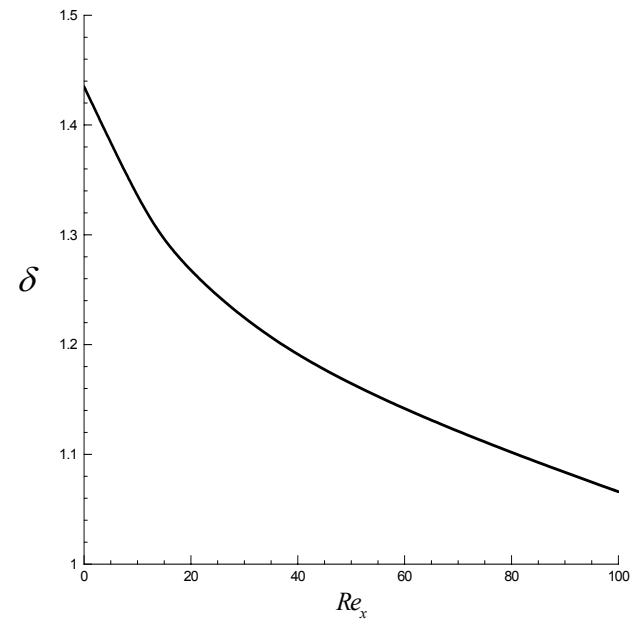

Fig. 7. Variation of (dimensionless) boundary layer thickness in the $\mathrm{x}$-direction (represented by $\mathrm{Re}_{\mathrm{x}}$ ) for a Giesekus fluid in plane stagnation flow $(\alpha=0.25$ and $W i=0.3)$.

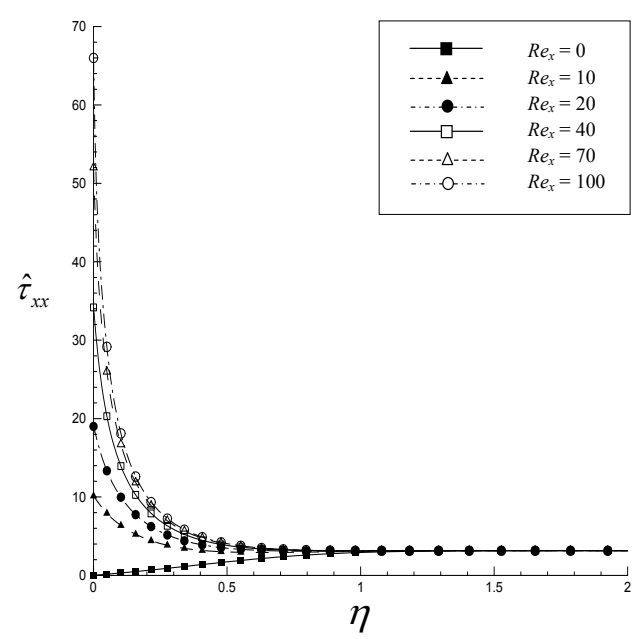

Fig. 8. Variation of the primary normal stress, $\hat{\tau}_{x x}$, in the x-direction (represented by $\mathrm{Re}_{\mathrm{x}}$ ) for a Giesekus fluid in plane stagnation flow $(\alpha=0.25$ and $\mathrm{Wi}=0.3)$. 
but, as previously mentioned, physical significance should be given only to the results obtained for $\alpha \leq 0.5$. From Fig. 11 it can be inferred that by an increase in the mobility factor the thickness of the boundary layer is decreased (see also Table II). The effect of the mobility factor on the boundary layer thickness is not surprising realizing the fact that the shear and extensional viscosities are both decreased by an increase in the mobility factor. A close look at Fig. 3 and Fig. 11 suggests that, the effect of the mobility factor on the velocity profiles is less significant when compared with the effect of the fluid's elasticity on them. This is not surprising realizing the fact that stagnation flows, by their very nature, are transient in a Lagrangian sense. That is to say that, the

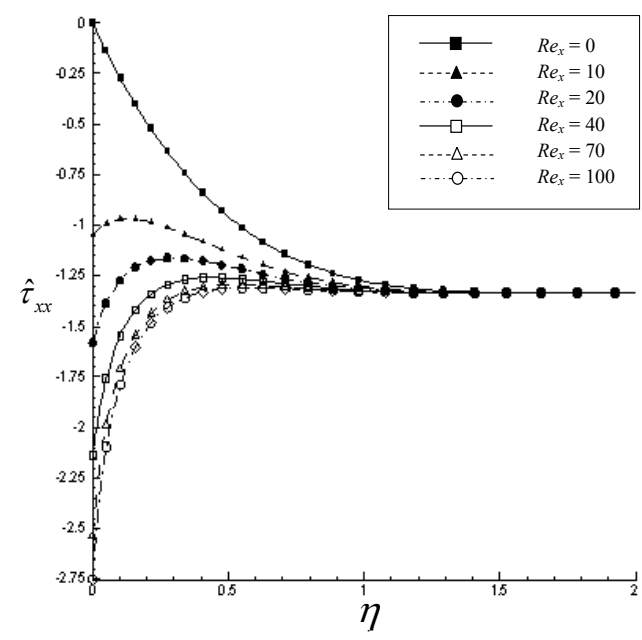

Fig. 9. Variation of the secondary normal stress, $\tau_{y y}$, in the x-direction (represented by $R e_{x}$ ) for a Giesekus fluid in plane stagnation flow $(\alpha=0.25$ and $W i=0.3)$.

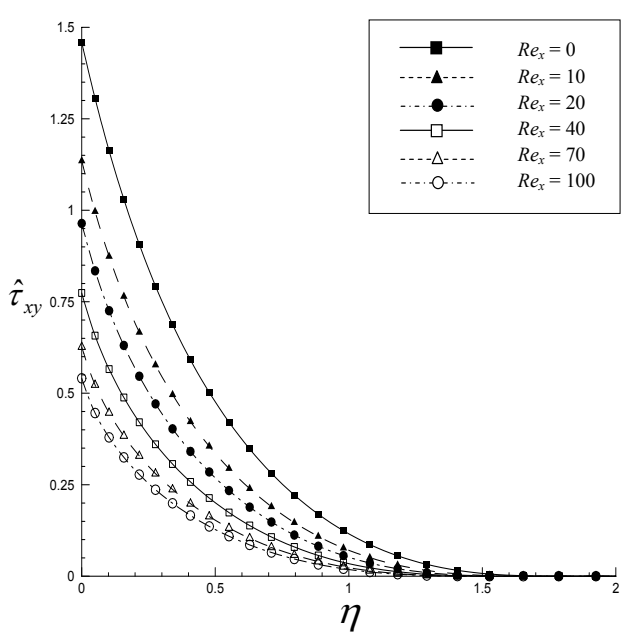

Fig. 10. Variation of the dimensionless shear stress profiles, $\hat{\tau}_{x y}$, in the $\mathrm{x}$-direction (represented by $\mathrm{Re}_{\mathrm{x}}$ ) for a Giesekus fluid in plane stagnation flow $(\alpha=0.25$ and $W i=0.3)$. strain history, or, more precisely, the residence time of fluid elements moving along different streamlines are not the same regardless of the fact that the flow is steady in an Eulerian sense. Obviously for those streamlines which are very close to the stagnation point the residence time is much longer so that the elastic stresses may indeed reach to high levels at the proximity of the stagnation point. In contrast, the effect of the mobility factor can be expected to become more pronounced by getting away from the stagnation point where shear and strain rates are larger thereby affecting the homogeneity of the relaxation time. Indeed our calculations show that the effect of the mobility factor becomes more pronounced when $\mathrm{x}$ is progressively increased (see Ref. 21 for more details) although we should stress that our computer code becomes unstable when $\mathrm{x}$ becomes too large.

\section{CONCLUDING REMARKS}

The exact solutions found by Phan-Thien in stagnation flows of Maxwell and Oldroyd-B fluids ${ }^{13,14)}$ cannot be applied to shear-thinning or shear-thickening fluids. Nor can it be of any good in flows where strong extensional deformations are predominant. The brand-new exact solution found in the present work for Giesekus fluids in plane stagnation flow remedies the shortcomings of the above-mentioned rheological models. Thus it can be argued to be a better tool for checking the performance of current or under development computer codes devised to be used for viscoelastic fluids. This exact solution also offers a more efficient tool for evaluating the validity of boundary layer theory for viscoelastic fluids. In

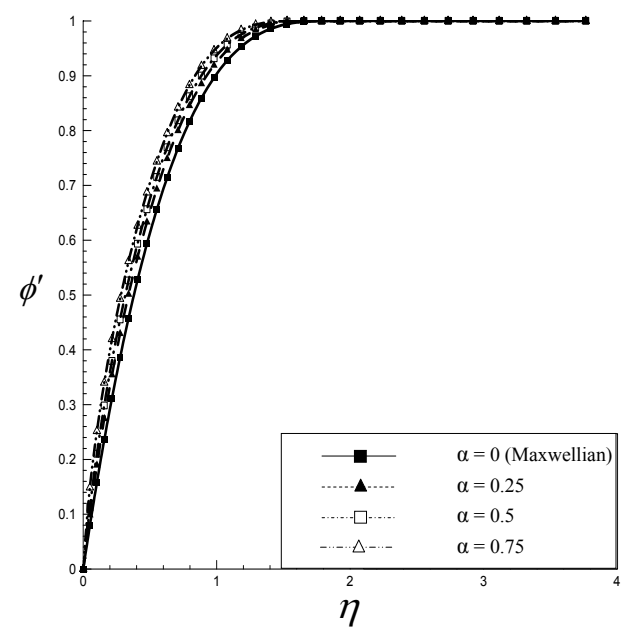

Fig. 11. Effect of the mobility factor on the velocity profiles for a Giesekus fluid in plane station flow $\left(R e_{x}=5\right.$ and $\left.W i=0.3\right)$. 
fact, it is found that for viscoelastic fluids the boundary layer becomes thinner the higher the Weissenberg number. More importantly, it is concluded that the thickness of the boundary layer formed near the wall is not a constant but a decreasing function of the distance from the stagnation point. This effect was attributed to the mobility factor and the fact that it controls the degree of shear-thinning and strain-thickening behavior of the fluid. Based on the fact that for purely-viscous shear-thinning fluids the boundary layer has a constant thickness, a non-uniform boundary layer found for Giesekus fluids can be attributed to the extensional nature of the fluid. It also suggests that for simulating flows of viscoelastic fluids in geometries in which fluid elements are subjected to strong elongational deformations (e.g., stagnation flows) constitutive equations with good performance in extensional flows should be used in the analysis.

\section{Acknowledgement}

The authors wish to thank the reviewers for their constructive comments.

\section{REFERENCES}

1) Tanner RI, Physics of Fluids, 9, 1246 (1966).

2) Rajagopal KR, J. of Non-Newtonian Fluid. Mech., 15, 239 (1984).

3) Davies MH, ZAMP, 17, 189 (1996).

4) Rajagopal KR, Gupta AS, Wineman AS, Appl. Sci. Eng. Lett., 18, 875 (1980).

5) Crochet MJ, Bezy M, "Numerical Simulation of NonNewtonian Flow", Elsevier, Amsterdam, (1984).
6) Owens RG, Philips TN, “Computational Rheology", Imperial College Press, London, (2002).

7) Garg VK, Rajagopal KR, Mech. Res. Commun., 17, 415 (1990).

8) Labropulu F, Husain I, Chinichian M, Int. J. Mathematics and Mathematical Sciences, 61, 3249 (2004).

9) Phan-Thien N, J. Non - Newtonian Fluid Mechanics, 13, 325 (1983).

10) Phan-Thien N, Dudek J, Boger DV, Tirtaatmadja V, J. NonNewtonian Fluid Mechanics, 18, 221 (1985).

11) Ji Z, Rajagopal KR, Szeri AZ, J. of Non-Newtonian Fluid Mechanics, 361 (1990).

12) Bhatnagar RK, Rajagopal KR, Gupta G, J. Non-Newton. Fluid. Mech., 4649 (1993).

13) Phan-Thien N, Rheol Acta, 22, 127 (1983).

14) Phan-Thien N, Rheol Acta, 23, 172 (1984).

15) Sadeghy K, Hajibeygi H, Taghavi SM, Int. J. Non-Linear Mech., 41, 1242 (2006).

16) Bird RB, Armstrong RC, Hassager O, "Dynamics of Polymeric Liquids", Vols. 1 and 2, 2nd ed., Wiley, New York, (1987).

17) Larson RG, "Constitutive Equations for Polymer Melts and Solutions”, Butterworths, Boston, (1988).

18) Gorla RSR, Dakappagari V, Int. J. Heat and Fluid Flow, 14, 408 (1983).

19) Keller HB, "Numerical Methods for Two-Point Boundary Value Problems", Ginn-Blaisdell, Waltham, Massachusetts, (1970).

20) Schichting H, "Boundary Layer Theory", $6^{\text {th }}$ Edition, McGrawHill, New York, USA, (1964).

21) Mirzadeh M, "Stagnation-Point Flow of Giesekus Fluids", B.Sc. Thesis, University of Tehran, Dept. of Mech. Eng, Tehran, Iran, 2008 